\title{
Mapping the ruminant microbiome across the gastrointestinal tract
}

Fei Xie

Wei Jin

Huazhe Si

Yuan Yuan

Ye Tao

Junhua Liu

Xiaoxu Wang

Chengjian Yang

Qiushuang Li

Xiaoting Yan

Limei Lin

Qian Jiang

Lei Zhang

Changzheng Guo

Chris Greening

Rasmus Heller

Leluo Guan

Phillip B. Pope

Zhiliang Tan

Weiyun Zhu

Min Wang

Qiang Qiu

Zhipeng Li

Shengyong Mao

\section{Video Byte}

Keywords: Microbiome, ruminant, gastrointestinal microbiome, metagenomics, metagenome-assembled genomes, Alphaproteobacteria, feed efficiency, methane production

Posted Date: October 13th, 2021

DOI: https://doi.org/10.21203/rs.3.rs-967565/v1 
License: (c) (i) This work is licensed under a Creative Commons Attribution 4.0 International License. Read Full License 


\section{Abstract}

Ruminants have long been important in agriculture due to their ability to convert fibrous plant substrates into foods we can digest, like milk and meat. This unique digestive ability is directly tied to their gastrointestinal microbiome, which has made their microbiome a subject of interest to nutritionists, microbiologists, and physiologists. However, most microbiome research to date has focused on the rumen, and we lack a comprehensive map of the microbial diversity throughout their gastrointestinal tract (GIT). A recent study characterized the microbiome across the GIT by sampling 10 GIT regions in 7 ruminant species. In addition to common agricultural species, they sampled water buffalo, yak, roe deer, and water deer. Using this data, they constructed a microbial reference catalog with over 154 million nonredundant genes and over 10,000 metagenome-assembled genomes. They identified 8,745 uncultured candidate species, which encode genes that play key roles in ruminant digestion. Future research can use this catalog to explore livestock production questions about feed efficiency, methane production, and overall ruminant health. 